\title{
Characterization of GRK5 as a novel regulator of rhabdomyosarcoma tumor cell growth and self-renewal
}

\author{
Thao Pham ${ }^{1}$, Kristin Robinson ${ }^{1}$, Terra Vleeshouwer-Neumann ${ }^{1}$, James E. Annis ${ }^{2}$ \\ and Eleanor Y. Chen ${ }^{1}$ \\ ${ }^{1}$ Department of Pathology, University of Washington, Seattle, WA, USA \\ ${ }^{2}$ Quellos HTS Core, Institute for Stem Cell and Regenerative Medicine, University of Washington, Seattle, WA, USA \\ Correspondence to: Eleanor Y. Chen, email: eleanor2@uw.edu \\ Keywords: rhabdomyosarcoma; self-renewal; GRK5, kinase; cell cycle
}

Received: December 05, $2019 \quad$ Accepted: April 03, $2020 \quad$ Published: April 21, 2020

Copyright: Pham et al. This is an open-access article distributed under the terms of the Creative Commons Attribution License 3.0 ( $C$ C BY 3.0), which permits unrestricted use, distribution, and reproduction in any medium, provided the original author and source are credited.

\section{ABSTRACT}

\begin{abstract}
Rhabdomyosarcoma (RMS) is the most common soft-tissue pediatric sarcoma. Clinical outcomes for RMS patients with relapsed or metastatic disease remain poor. Treatment options remain limited, presenting an urgent need for novel therapeutic targets. Using a high-throughput siRNA screen against the human kinome, we identified GRK5, a G-protein receptor kinase, as a novel regulator of RMS tumor cell growth and self-renewal. Through functional assays in vitro and in vivo, we show that GRK5 regulates cell cycle in a kinase-independent manner to promote RMS tumor cell growth. NFAT1 expression is regulated by GRK5 in a kinase independent manner, and loss of NFAT1 phenocopies GRK5 loss-of-function effects on the cell cycle alterations. Self-renewal of tumor propagating cells (TPCS) is thought to give rise to tumor relapse. We show that loss of GRK5 results in a significant reduction of RMS self-renewal capacity in part due to increased cell death. Treatment of human RMS xenografts in mice with CCG-215022, a GRK5-selective inhibitor, results in reduced tumor growth and self-renewal in both major subtypes of RMS. GRK5 represents a novel therapeutic target for the treatment of RMS.
\end{abstract}

\section{INTRODUCTION}

Rhabdomyosarcoma (RMS) is the most common pediatric soft-tissue cancer. There are two major subtypes of RMS, each with distinct histologic features and genetic alterations. Embryonal rhabdomyosarcoma (ERMS) typically harbors mutations in the $R A S$ pathway [1]. Alveolar rhabdomyosarcoma (ARMS) is characterized by the presence of the PAX3- or PAX7-FOXO1 fusion [2]. Other RMS subtypes include spindle cell $(\mathrm{SC} / \mathrm{S})$, pleomorphic (PRMS) and those not otherwise specified (NOS). While the prognosis is good for patients with localized disease, the survival rate for patients with relapsed RMS is only 10-30\% [3], highlighting an urgent need for more effective treatment options for disease relapse. Tumor propagating cells (TPCs) are thought to be responsible for metastasis and relapse of some cancer types, such as breast and lung cancer [4-7], and possess stem cell-like characteristics that allow for the recapitulation of tumor heterogeneity in its entirety [7]. A potential TPC population with self-renewal capacity has been identified in a conserved transgenic zebrafish model of ERMS [8]. In human ERMS, CD133-positive cells have also been found to possess stem-like characteristics and are resistant to standard-of-care chemotherapy [9]. Targeting stem-like features of RMS would therefore provide novel therapeutic avenues for treating RMS disease relapse and metastasis.

Therapeutic targeting of protein kinases has been demonstrated to be an effective treatment option for a variety of cancers [10]. There exists at least 500 kinases in the human genome, many of which have been linked to the promotion of cancer progression and relapse $[10,11]$. The roles of kinases in the pathogenesis 
of cancer and other human diseases have been studied extensively over the past 20 years [12]. However, there currently exists only 48 FDA-approved kinase inhibitors, many of which share the same targets [12]. Of the 48 FDA-approved kinase inhibitors, none have been tested for their therapeutic effects against advanced RMS disease [12]. While previous studies have shown MEK, CDK4/6 and WEE1 as promising kinase targets for inhibiting tumor growth, druggable kinases against RMS self-renewal have been poorly characterized [13, 14]. The study by Chen et al (2014) shows that chemical inhibition of glycogen synthase kinase 3 (GSK3) reduces ERMS tumor growth and self-renewal, demonstrating the therapeutic potential for targeting protein kinases that play a role in the regulation of RMS tumor growth and self-renewal [15].

G-protein coupled receptor kinase 5 (GRK5) belongs to a family of serine/threonine kinases [16] and plays an important role in cardiovascular disease pathogenesis and early heart development [17-19]. GRK5 targets the $\beta$-adrenergic receptors, members of the G-protein coupled receptors family (GPCRs), leading to their desensitization and down regulation in cardiomyocytes [20], and is upregulated during heart failure [21]. GRK5 can also function in a non-GPCR-dependent manner to regulate HDAC5 activity in cardiomyocytes, promoting maladaptive hypertrophy and heart failure [22]. While GRK5 has been extensively studied for its role in heart disease, the role of GRK5 in cancer pathogenesis is poorly characterized. To date, GRK5 has been shown to play a role in the pathogenesis of lung, brain and prostate cancer [4, 23, 24]. In non-small cell lung cancer (NSCLC) and glioblastoma multiforme (GMB), GRK5 is highly expressed in primary patient specimens and depletion of GRK5 results in reduced cell growth [4, 23]. Loss of GRK5 in NSCLC and prostate cancer cell lines also results in cell cycle arrest $[23,24]$. However, the role of GRK5 in RMS pathogenesis is unknown. GRK5 possesses a unique combination of kinase activity and non-enzymatic protein domains for interacting with substrates, making it an attractive target for drug design in translational applications [20, 25, 26].

In this study, we have identified GRK5 as a novel regulator of RMS self-renewal in a high-throughput siRNA library screen against the human kinome (714 kinases). Using the CRISPR/Cas9-based genetic editing strategy, we show that GRK5 loss-of-function reduces RMS selfrenewal capacity in vitro and in vivo in part through increased programmed cell death. GRK5 regulates cell cycle progression to promote ERMS tumor cell growth in a kinaseindependent manner. NFAT1, a transcription factor involved in T-cell maturation [27], is a key player in GRK5-mediated cell cycle progression. Treatment of RMS xenografts with a selective GRK5 inhibitor, CCG-215022, results in a significant reduction of tumor growth, demonstrating the potential of GRK5 as a therapeutic target in RMS.

\section{RESULTS}

\section{A siRNA library screen of the human kinome identifies GRK5 as a novel regulator of ERMS self-renewal}

To identify potential candidate kinases that are essential for self-renewal of ERMS, we performed a siRNA library screen against the human kinome (714 kinases) in two ERMS cell lines (RD and 381T). Each cell line was transfected with a pool of 3 siRNAs against each kinase, along with control (scramble) siRNAs, in 384-well low attachment plates to induce sphere formation. The sphere assay was used as a surrogate in vitro assay for assessing the self-renewal capacity of tumor cells [28]. RD and $381 \mathrm{~T}$ cells were also transfected with the same set of siRNAs in adherent conditions for assessing cell growth. An ATP-based viability assay was performed on siRNAtransfected cells in adherent condition, and high-content imaging was performed on the spheres. The normalized ratio of self-renewal capacity to cell growth compared to controls for each kinase target was analyzed (see the volcano plot in Figure 1A). Of the 714 kinases screened, 6 top candidate genes (FES, LTK, LYN, NME9, PIK3C2A, $G R K 5)$ showed differential effects on self-renewal compared to cell growth and were prioritized for further validation. We subsequently utilized a high-efficiency CRISPR/Cas9 gene targeting strategy [29] to validate the loss-of-function effect of each candidate kinase gene on self-renewal of $381 \mathrm{~T}$ ERMS cells (Figure 1B). GRK5 was prioritized for further functional characterization due to consistent loss-of-function effects on self-renewal of RMS cells.

\section{GRK5 is differentially expressed in RMS cells compared to normal tissue types and is present in both nuclear and cytoplasmic compartments}

GRK5 mRNA expression levels were analyzed in 4 RMS cell lines (381T and SMS-CTR of the ERMS subtype; Rh5 and Rh30 of the ARMS subtype) and compared against a primary myoblast line and an immortalized fibroblast line. In the 4 RMS cell lines, regardless of subtype, the expression level of GRK5 is at least 2-fold higher compared to normal cell types (Figure 1C). Immunofluorescence showed both nuclear and cytoplasmic localization of GRK5 in RMS cells (Figure 1D). Immunohistochemistry performed on a tissue microarray (TMA) of primary human RMS tumors showed positive GRK5 expression in the majority of RMS samples including 8/10 ERMS and 10/17 ARMS samples (Figure 1E). In contrast, normal muscle samples from 4 patients showed very weak or negative GRK5 expression. From these findings, GRK5 appears to be differentially expressed in RMS tumors and likely plays an important role in RMS pathogenesis. 


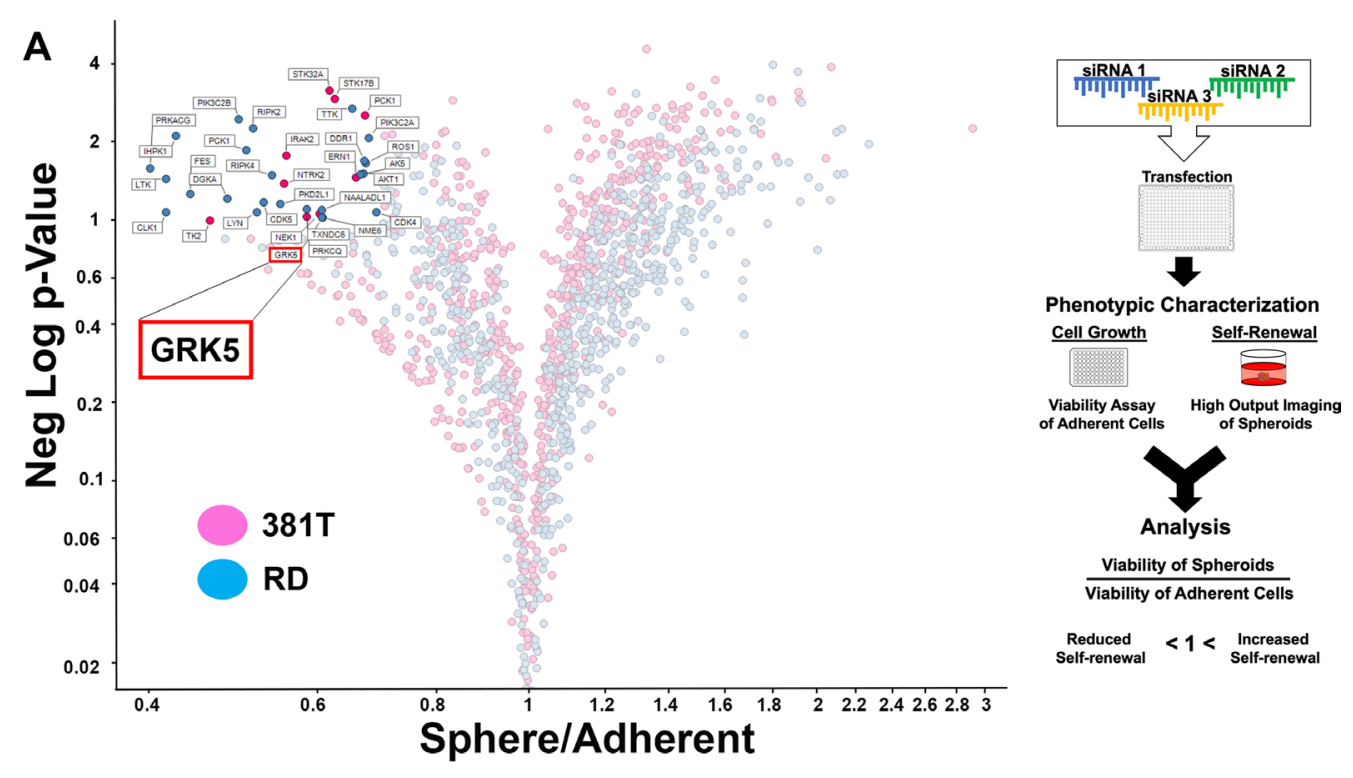

B

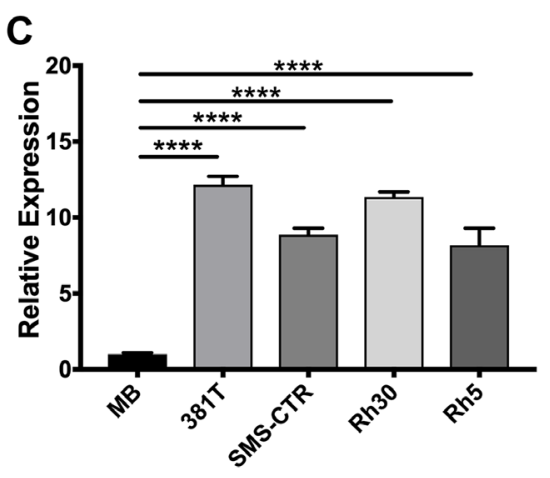

D
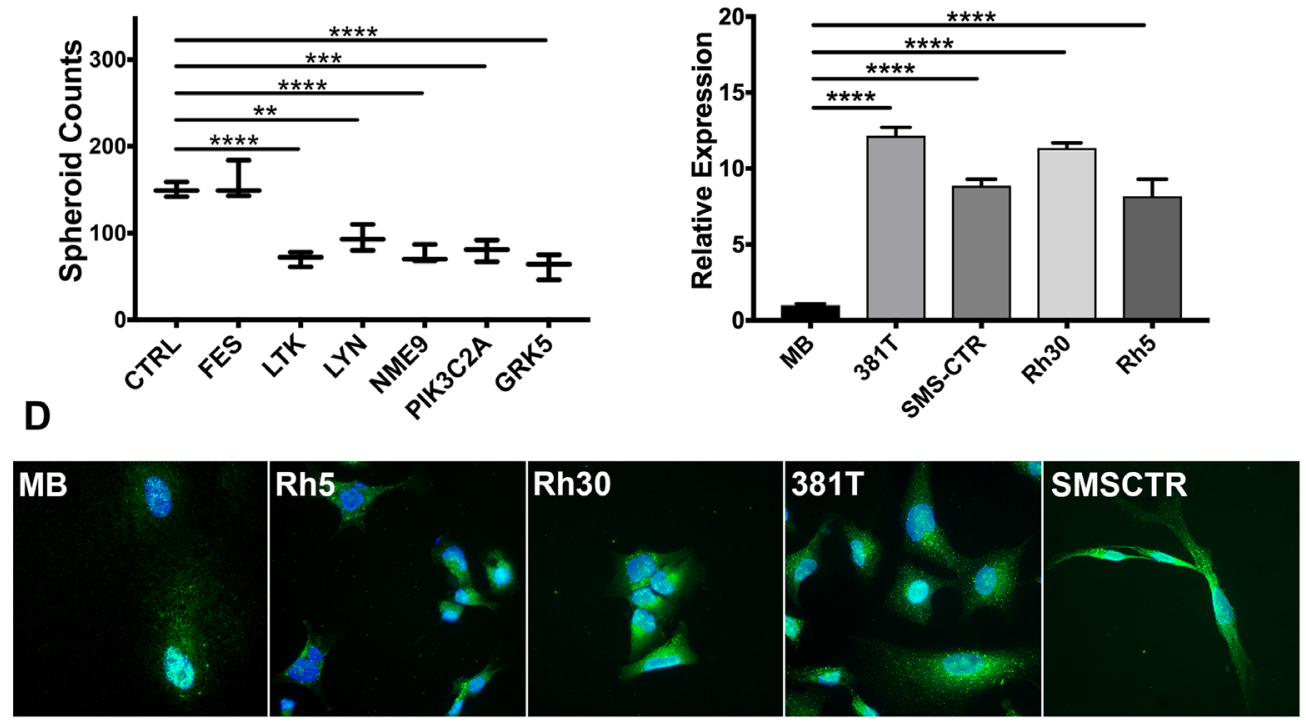

E
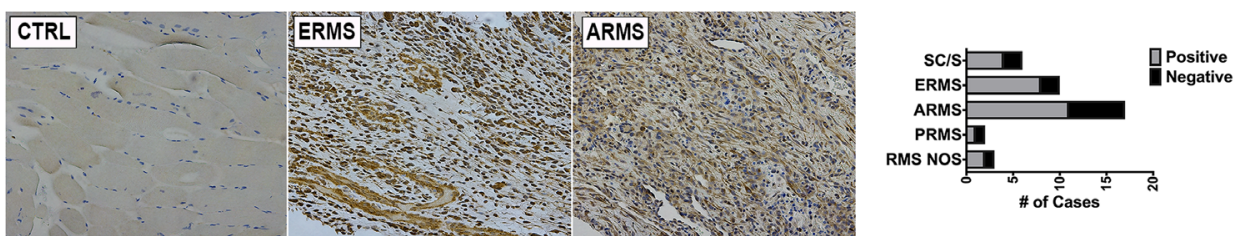

Figure 1: An siRNA library screen of the human kinome identifies GRK5 as a novel regulator of ERMS self-renewal. (A) Volcano plot illustrating candidate kinases identified from an siRNA library screen against the human kinome in ERMS cancer cell lines (381T and RD). Significant hits are indicated as having a $p$-value (Y-axis) of $<0.05$ and a Sphere/Adherent viability ratio (X-axis) of $<1.0$. Diagram on the right illustrates workflow and analysis used in the siRNA library screen. GRK5 is highlighted as being a candidate kinase identified from the screen. (B) Spheroid counts to assess self-renewal capacity was performed on CRISPR/Cas9 mediated knockout of top 6 candidate kinases (FES, LTK, LYN, NME9, PIK3C2A, GRK5). Error bars represent standard deviation of 3 technical replicates from an individual experiment that was repeated 3 times. (C) RT-PCR analysis of GRK5 expression in human myoblasts (MB) compared to a panel of RMS cancer cell lines (381T, SMS-CTR, RH30, RH5). Error bars represent standard deviation of 3 technical replicates from an individual experiment that was repeated 3 times. (D) Immunofluorescence images showing GRK5 staining in MB and RMS cancer cell lines (381T, SMS-CTR, Rh30, Rh5). (E) Immunohistochemistry of GRK5 in skeletal muscle control (CTRL) and representative primary ERMS and ARMS tumors. Summary of IHC for GRK5 in primary RMS tumors spotted on a tissue microarray is shown on the right. Spindle cell RMS (SC/S), embryonal RMS (ERMS), alveolar RMS (ARMS), pleomorphic RMS (PRMS), RMS not otherwise specified (RMS NOS). Two-tailed $t$-test; ${ }^{* *}=p<0.01 ;{ }^{* * *}=p<0.001,{ }^{* * * *}=p<0.0001$. 


\section{GRK5 regulates self-renewal of both ERMS and ARMS}

To confirm effective disruption of GRK5 by CRISPR/Cas9, gRNAs were designed to flank the catalytic, nuclear export (NES) and nuclear localization (NLS) functional domains of GRK5 (Figure 2A). Genetic disruption of GRK5 was then verified via PCR amplification of the genomic deletion event, and depletion of the protein product was confirmed by Western blots (Figure 2A, 2B, Supplementary Figure 1A). Immunofluorescence of SMS-CTR GRK5-targeted cells showed reduced GRK5 signal, highlighting specificity of gene targeting (Supplementary Figure 1B). We assessed the loss-of-function effect of GRK5 on tumor cell selfrenewal using the sphere assay on a panel of ARMS (Rh5 and Rh30) and ERMS (381T and SMS-CTR) cell lines. CRISPR/Cas9-mediated disruption of GRK5 resulted in a significant reduction ( $\mathrm{p}$-value $<0.05$ ) in the sphere formation in 3 (381T, SMS-CTR, Rh5) out of the 4 RMS cancer cell lines (Figure 2C). Targeted disruption of GRK5 in Rh30 showed a trend of reduced self-renewal capacity but was not statistically significant $(p$-value $=0.086$ ) Spheroids generated from cells harboring targeted disruption of GRK5 appeared to be both smaller and fewer in number (Figure 2C, 2D, Supplementary Figure 1C). To determine the cellular mechanism underlying the lossof-function effects of GRK5 on sphere formation, we performed a quantitative flow cytometry-based Annexin V assay to assess for any change in apoptosis. Loss of GRK5 resulted in a significant increase in early apoptotic events in the spheroids (Figure 2E, 2F), and approximately 2-fold increase in total apoptotic cells (Figure 2G) compared to the controls. Increased in cell death in GRK5-deficient spheroid cells was further supported by elevated levels of cleaved caspase $3(\mathrm{CC} 3)$ protein (Figure $2 \mathrm{H})$. To assess the effects of GRK5 loss-of-function on the self-renewal capacity of RMS cells in vivo, we performed limiting dilution experiments of ERMS (381T) and ARMS (Rh5) xenografts in immunocompromised NOD-scidIL2Rgammanull (NSG) mice. In both RMS subtypes, targeted disruption of GRK5 resulted in approximately 4 to 6-fold reduction in self-renewal frequency (Table 1). Taken together, our results indicate that loss of GRK5 in RMS cells results in decreased self-renewal capacity in part through induction of programmed cell death.

\section{GRK5 regulates ERMS cell growth in a kinase-independent manner and is involved in regulating cell cycle progression}

We assessed the loss-of-function effect of GRK5 on tumor cell growth using an ATP-based viability assay on a panel of ARMS (Rh5 and Rh30) and ERMS (381T and SMS-CTR) cell lines. Loss of GRK5 resulted in a significant reduction in cell viability in all 4 RMS cell lines ( $p$-value $<0.05)$ (Figure 3A). To assess the specificity of GRK5 loss-of-function effect on RMS cell growth, we overexpressed a Cas9-resistant form of GRK5 in the presence of CRISPR/Cas9-mediated GRK5 gene disruption in SMS-CTR cells. Compared to the GFP overexpression control, Cas9-resistant GRK5 rescued the growth phenotype of SMS-CTR cells following targeted disruption of GRK5 (Figure 3B). Even though the GRK family proteins are known for their kinase-dependent roles, some studies have also implicated kinaseindependent function of GRK5 [20, 30]. To determine whether GRK5 regulates RMS cell growth in a kinase dependent or independent manner, we generated a kinase dead (K215R) (KD) GRK5 mutant [25] that is resistant to targeted disruption by CRISPR/Cas9. Overexpression of KD GRK5 protein also restored cell growth in SMSCTR cells with targeted disruption of GRK5 (Figure 3B), indicating that GRK5 regulates ERMS cell growth in a kinase-independent manner. We next determined the cellular event that was responsible for GRK5 loss-offunction effect on RMS cell growth. While 381T and SMS-CTR cells with targeted disruption of GRK5 showed no significant change in cellular differentiation or cell death (Figure 3C, 3D), they showed altered cell cycle progression in a flow cytometry-based cell cycle analysis following EdU pulse by day 6 of GRK5 knockout (Supplementary Figure 2A). This delay in cell cycle progression is sustained till day 10 with a delay in the G1/S phase in 381T cells, and in the G2/M phase in SMSCTR cells (Figure 3E, 3F). Overall, our results indicate that GRK5 functions in a kinase-independent manner to alter cell cycle progression in ERMS cells.

\section{NFAT1 expression is regulated by GRK5 in a kinase-independent manner}

GRK5 has been previously shown to facilitate the transcriptional activity of NFAT as part of a DNA binding complex during cardiac hypertrophy in a kinase independent manner [20]. NFAT1 has been shown to function either as a positive or negative regulator of cell cycle progression [31,32]. To investigate the role of NFAT as a potential downstream component of the GRK5 pathway in regulating ERMS cell growth, we first showed that targeted disruption of GRK5 led to decreased levels of NFAT1 expression in 381T and SMS-CTR cells (Figure 4A). Overexpression of wild-type or catalyticallydead GRK5 restored expression levels of NFAT1 (Figure 4B). To determine the loss-of-function effects of NFAT1 on RMS cells, we showed that targeted disruption of NFAT1 by CRISPR/Cas9 in ERMS cells (381T and SMS-CTR) significantly reduced cell growth (Figure 4C). Cell cycle analysis of NFAT1-targeted $381 \mathrm{~T}$ and SMS-CTR cells showed significant alteration of cell cycle progression (Figure 4D, 4E). There was a delay in both G1/S and G2/M in NFAT1-disrupted 381T and SMS-CTR cells. To explore 

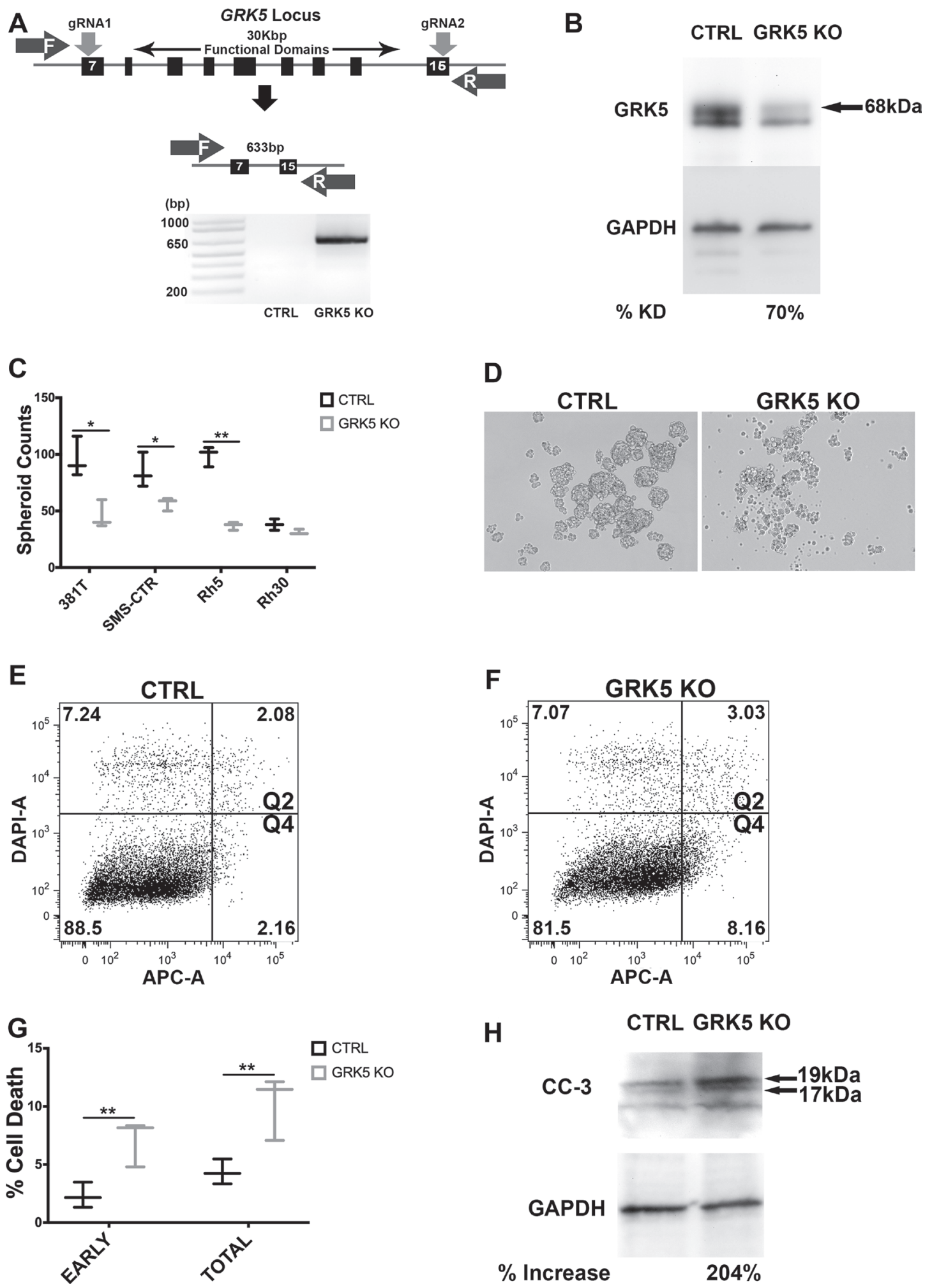

Figure 2: GRK5 regulates self-renewal of both ERMS and ARMS. (A) Schematic demonstrating CRISPR/Cas9 targeting of GRK5. Two gRNAs were designed to flank the region encoding functional domains of GRK5. Genetic disruption was confirmed via PCR amplification of the deleted GRK5 domain followed by Western analysis of depleted protein (B). Spheroid counts to assess self-renewal (C). Representative images (D) of control (CTRL) and GRK5 knockout (GRK5 KO) spheroids are from 381T cells. Results shown are of 3 replicates from one of 3 independent experiments. (E-G) Annexin V Flow cytometry to assess the extent of apoptosis (quadrants 2 and 4 ) in SMS-CTR GRK5 knockout spheroid cells (GRK5 KO) compared to Cas9 only controls (CTRL). (H) Western analysis of cleaved Caspase 3 (CC3) comparing SMS-CTR GRK5 KO spheroids to controls (CTRL). Error bar represents standard deviation and two-tailed t-test $(\mathrm{C}, \mathrm{G})$ was performed on 3 replicates from one of three independent experiments. ${ }^{*}=p<0.05 ;^{* *}=p<0.01$. 
Table 1: Summary of limiting dilution experiments

\begin{tabular}{|c|c|c|c|c|c|}
\hline \multicolumn{3}{|l|}{$381 \mathrm{~T}$} & \multicolumn{3}{|l|}{ Rh5 } \\
\hline Cell No. & Control & GRK5 KO & Cell No. & Control & GRK5 KO \\
\hline 50,000 & 6 of 6 & 6 of 6 & 50,000 & 6 of 6 & 6 of 6 \\
\hline 10,000 & 6 of 6 & 5 of 6 & 10,000 & 6 of 6 & 4 of 6 \\
\hline 2,000 & 5 of 6 & 3 of 6 & 2,000 & 5 of 6 & 3 of 6 \\
\hline TPC frequency & 1115 & $4373^{*}$ & TPC frequency & 1115 & $6230^{* *}$ \\
\hline $95 \% \mathrm{CI}$ & 414-3002 & 1927-9922 & $95 \% \mathrm{CI}$ & 414-3002 & 2731-14214 \\
\hline${ }^{*} p=0.0353$ & & & ${ }^{* *} p=0.00739$ & & \\
\hline
\end{tabular}

$381 \mathrm{~T}$ and Rh5 RMS cells were injected into NSG mice at limiting dilutions of $2 \times 10^{3}, 1 \times 10^{4}, 5 \times 10^{4}$ cells. TPC frequency was calculated as previously described [43]. Confidence interval and statistics was performed on an $\mathrm{n}=6 \mathrm{mice}$ per control and GRK5 KO. ${ }^{*}=p<0.05 ;^{* *}=p<0.01$.

whether GRK5 interacts with NFAT1 in ERMS, we showed by immunofluorescence that GRK5 and NFAT1 appear to be co-localized in $381 \mathrm{~T}$ cells (Figure 4F). By proximity ligation assay, we showed GRK5 and NFAT1 in close proximity in the nucleus and the cytoplasm of $381 \mathrm{~T}$ cells, and that loss of GRK5 abrogated this interaction (Figure 4G). Taken together, our data indicate that NFAT1 is a potential downstream mediator of GRK5 function in regulating RMS cell growth.

\section{Treatment of RMS tumor with CCG-215022, a GRK5 inhibitor, reduces tumor growth in vivo}

To assess the potential of GRK5 as a therapeutic target against RMS, 381T or Rh5 RMS xenografts established in NSG mice were treated with a GRK5selective inhibitor, CCG-215022 (Figure 5A). Treatment duration (21 days for $381 \mathrm{~T}$ tumors and 12 days for Rh5 tumors) was determined by how quickly DMSO vehicle control (CTRL)-treated tumors reached experimental endpoint. Mice treated with CCG-215022 appeared to show delayed $381 \mathrm{~T}$ and Rh5 tumor growth over time compared to mice treated with vehicle control (Figure 5B, 5C). CCG-215022 treated tumors showed a significant reduction in tumor growth in both $381 \mathrm{~T}$ and Rh5 tumors compared to the mice treated with vehicle control (Figure 5D-5F). Immunohistochemistry analysis of CCG-215022 treated tumors shows a lower Ki-67 proliferation index compared to the vehicle controltreated tumors (Figure 5G). These results demonstrate the therapeutic potential of targeting GRK5 as an alternative treatment option to inhibit RMS tumor growth.

\section{DISCUSSION}

While GRK5 has been extensively studied for its role in the pathogenesis of cardiovascular disease, only in recent years has its role in cancer biology been brought to light [17-22]. GRK5 has been implicated as having a role in regulating lung, brain and prostate cancer cell growth [4, 23, 24]. However, the role of GRK5 in
RMS has yet to be investigated. In this study, we show that GRK5 is a novel regulator of RMS cell growth through its interaction with NFAT1 to regulate cell cycle progression in a kinase-independent manner. Loss of GRK5 reduces the self-renewal capacity of RMS cells through increased programmed cell death, implicating it as a novel regulator of stem-like features in RMS. We demonstrate the potential for GRK5 as a therapeutic target through treatment of RMS xenografts with a selective GRK5 inhibitor, CCG-215022. RMS tumors treated with CCG-215022 show significant reduction in tumor growth and self-renewal capacity.

The studies characterizing the role of GRK5 in cancer pathogenesis to date have primarily investigated the functional requirement of its kinase activity [33, 34]. TP53 phosphorylation by GRK5 leads to its degradation, resulting in inhibition of the TP53-dependent apoptotic response to genotoxicity in osteosarcoma cells [33]. A study using HeLa cells additionally shows a defect in proper cell cycle progression following GRK5 gene knockdown [34]. However, we show through rescue experiments with a GRK5 kinase-dead mutant that GRK5 regulates RMS cell growth in a kinase-independent manner. We also show that targeted disruption of GRK5 in two RMS cell lines with TP53 mutations, 381T with TP53(R248W) and Rh30 with TP53(R237H) [35, 36], reduces RMS cell growth despite the presence of TP53 mutations. Our findings indicate that GRK5 regulates RMS cell growth in a kinase- and TP53-independent manner.

Dysregulated activity of the NFAT family of transcription factors has been identified in cancer [37]. While NFAT proteins (NFAT1-5) share similar DNA binding targets, each member possesses both redundant and opposing functions, and their activity often requires cooperation with additional transcriptional partners $[31,32,37]$. NFAT1 has been shown to function both as a tumor suppressor through the transcriptional activation of the CDK4 promoter or as an oncogene by silencing p15 expression [20]. In a model of pathological cardiac hypertrophy, GRK5 promotes the transcriptional activity 
of NFAT in a kinase-independent manner as part of a DNA binding complex to regulate expression of hypertrophic genes [20]. In this study, both wild-type and kinase-dead GRK5 are able to restore NFAT1 expression following targeted disruption of GRK5 in ERMS cell lines. We also show that GRK5 potentially interacts with NFAT1, and this interaction is abrogated with loss of GRK5. Our findings suggest a role for GRK5 acting in a kinase-independent manner to regulate the NFAT1 expression. CRISPR/Cas9 mediated-disruption of NFAT1 phenocopies the loss-offunction effects of GRK5 on cell cycle progression. NFAT likely functions as an important downstream mediator to promote oncogenic activity of GRK5. Further study is required to determine whether GRK5 directly or indirectly interacts with NFAT1 in ERMS in regulating ERMS tumor cell growth.

TPCs undergo self-renewal to recapitulate the complex heterogeneity of a given malignant tumor and

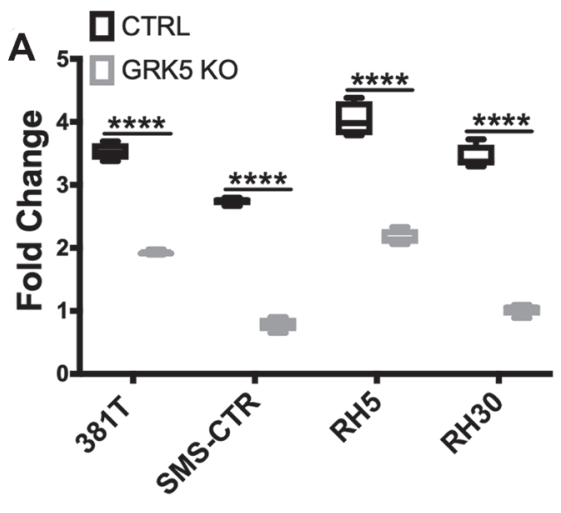

B
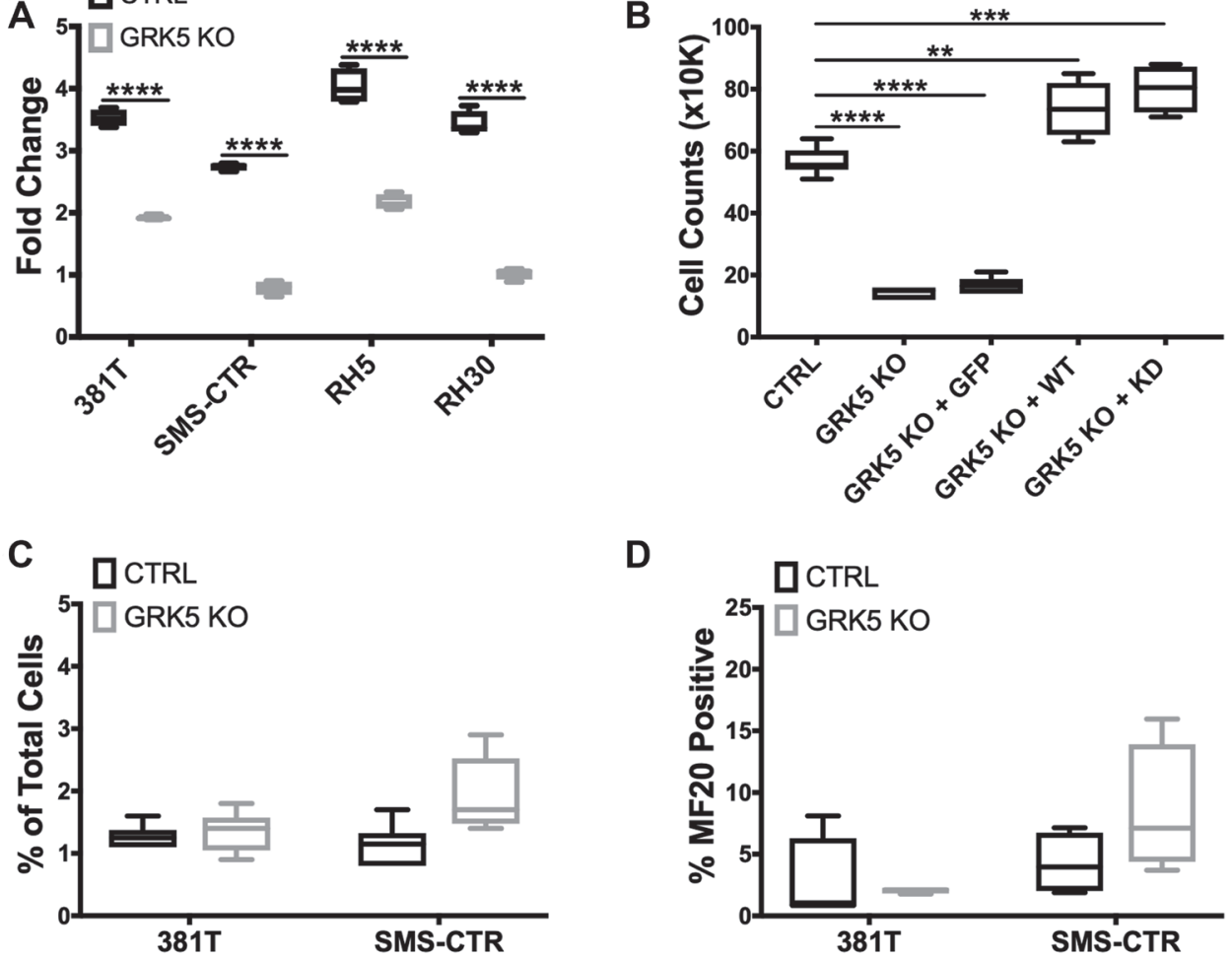

D
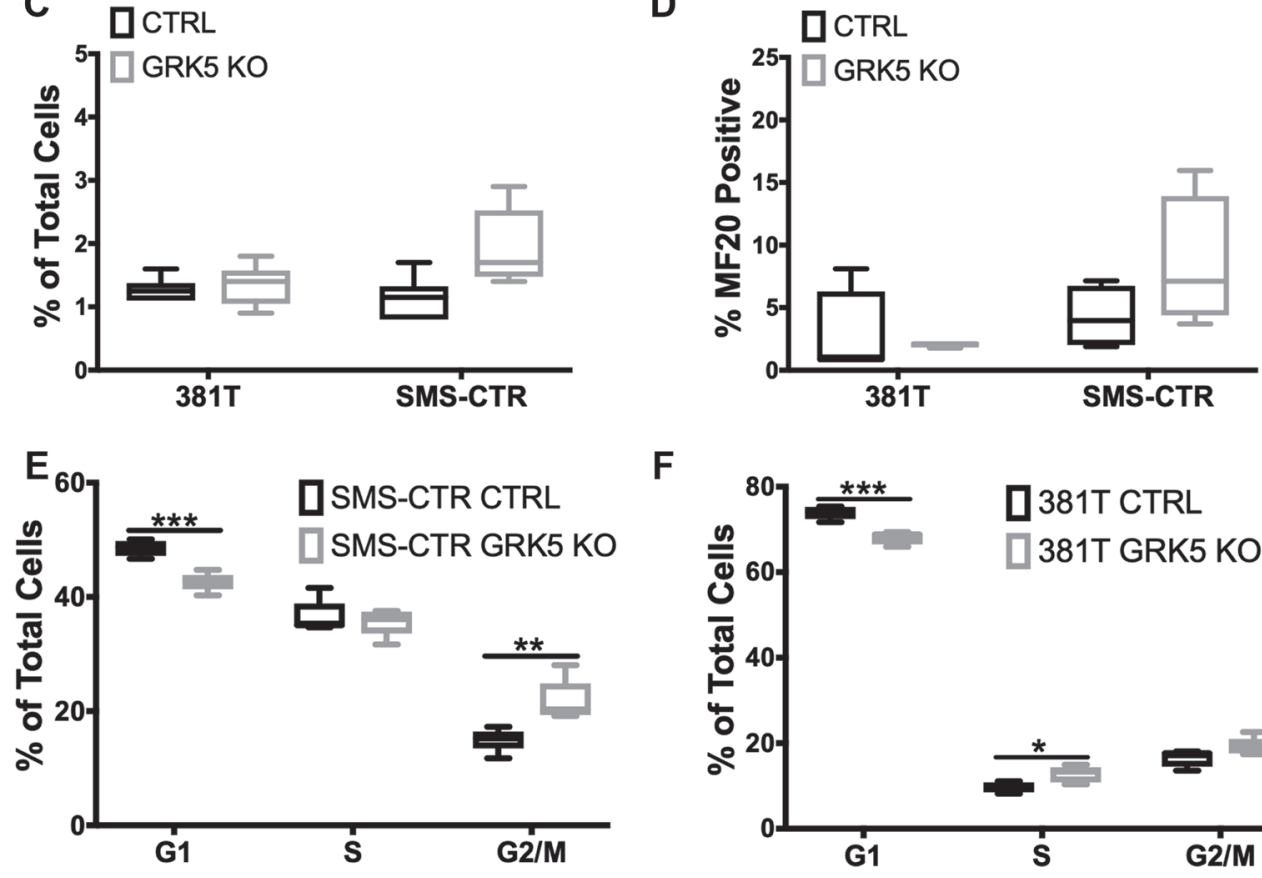

$\mathbf{F}$

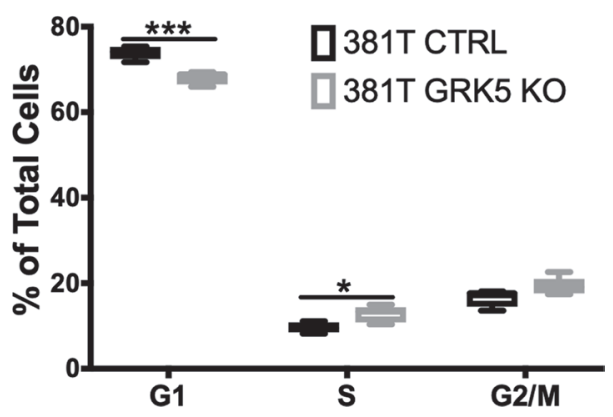

Figure 3: GRK5 regulates ERMS cell growth in a kinase-independent manner and is involved in regulating cell cycle progression. (A) Cell Titer Glo viability assessment of GRK5 knockout (GRK5 KO) compared to controls (CTRL) in a panel of RMS cancer cell lines (381T, SMS-CTR, Rh5, Rh30). Data shown are 4 replicates from one of 3 independent experiments. Two-tailed $t$-test, ${ }^{* * * *}=p<0.0001$. (B) Summary of cell count data from rescue experiment to demonstrate specificity of the GRK5 KO growth phenotype. SMS-CTR cells were transduced with lentivirus expressing either Cas9 only (CTRL), Cas9 with GRK5 gRNAs (GRK5 KO), Cas9 with GRK5 gRNAs and GFP overexpression (GRK5 KO + GFP), Cas9 with GRK5 gRNAs and Cas9 resistant, wildtype GRK5 (GRK5 KO + WT), Cas9 with GRK5 gRNAs and Cas9 resistant, kinase dead GRK5 (GRK5 KO + KD). Data shown are 6 replicates from one of three independent experiments. Two-tailed $t$-test, ${ }^{* *}=p<0.01,{ }^{* * *}=p<0.001,(\mathbf{C})$ Annexin V Flow cytometry to assess apoptosis. (D) Quantitation of immunofluorescence (IF) against MF20 in RMS cells with GRK5 knockout (GRK5 KO) and Cas9 only controls (CTRL). $(\mathbf{E}, \mathbf{F})$ EdU flow cytometry-based cell cycle analysis of SMS-CTR and 381T cells with Cas9 only as control (CTRL) or GRK5 knockout (GRK5 KO). Data shown are from 6 independent experiments. Two-tailed $t$-test, ${ }^{*}=p<0.05,{ }^{* *}=p<0.01,{ }^{* * *}=p<0.001,{ }^{* * * *}=p<0.0001$. 
A

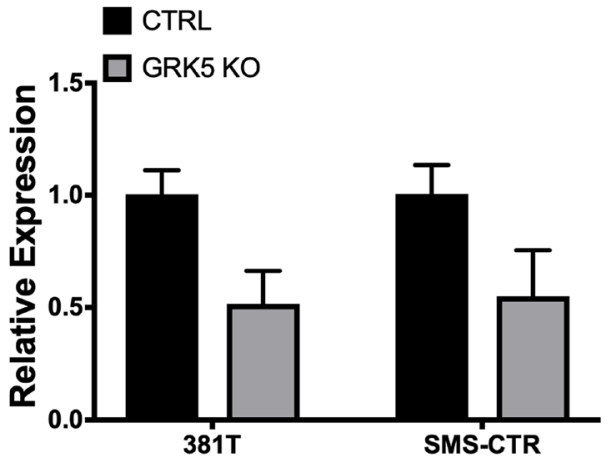

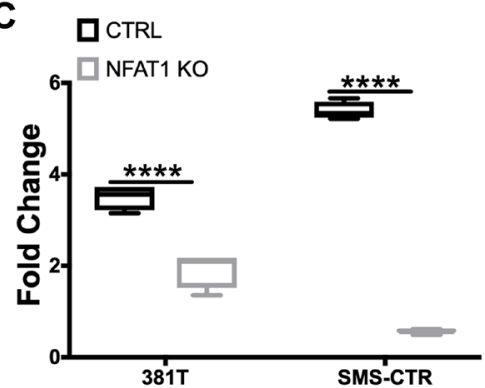

B

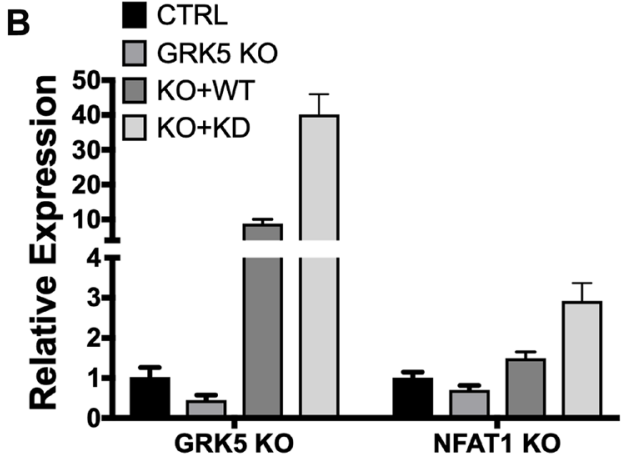

D DSMS-CTRCTRL

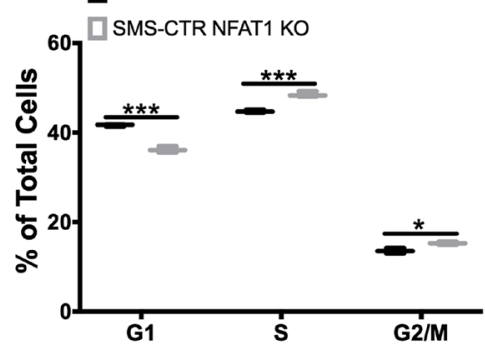

E

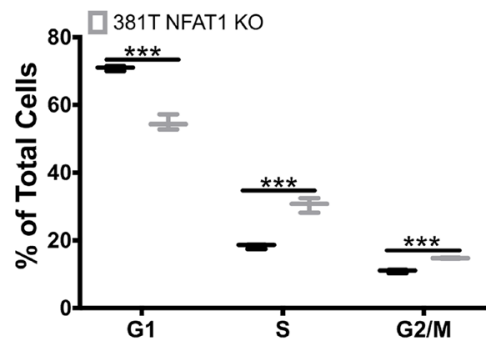

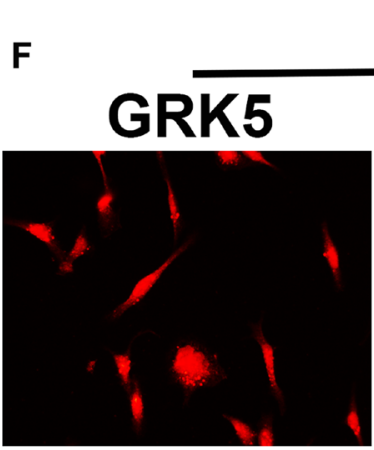

IF

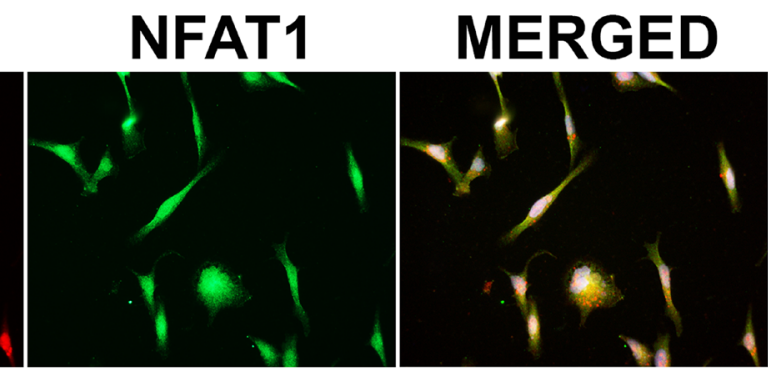

G

PLA

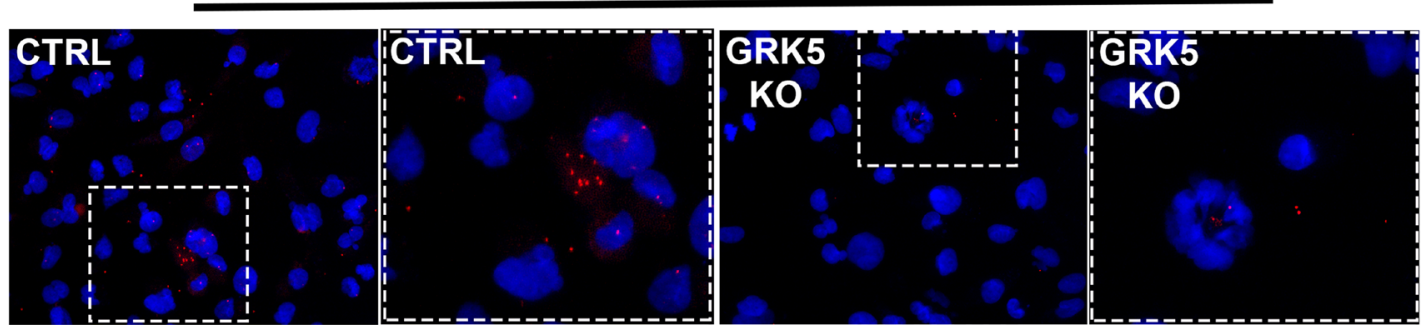

Figure 4: NFAT1 expression is regulated by GRK5 in a kinase-independent manner. (A) Quantitative RT-PCR analysis of NFAT1 expression in ERMS cells (381T and SMS-CTR) with Cas9 only control (CTRL) and GRK5 knockout (GRK5 KO). (B) RT-PCR analysis of GRK5 and NFAT1 expression in 381T cells following GRK5 rescue with wild-type and domain mutants. 381T cells were transduced with lentivirus expressing Cas9 only (CTRL), Cas9 with GRK5 gRNAs (GRK5 KO), Cas9 with GRK5 gRNAs and Cas9 resistant, wildtype GRK5 (GRK5 KO + WT), Cas9 with GRK5 gRNAs and Cas9 resistant, kinase dead GRK5 (GRK5 + KD). (C) Cell Titer Glo viability assessment of NFATl knockout (NFATl KO) compared to Cas9 only control (CTRL) in ERMS cancer cell lines (381T, SMS-CTR). Data shown are 4 replicates from one of 3 independent experiments, two-tailed $t$-test, ${ }^{* * * * *}=p<0.0001$. (D, E) EdU flow cytometry-based cell cycle analysis of 381T and SMS-CTR cells with Cas9 only as control (CTRL) or NFAT1 knockout (NFAT1 KO). Data shown are of 3 replicates from one of 3 independent experiments. Two-tailed $t$-test, ${ }^{*}=p<0.05,{ }^{* * *}=p<0.001$. (F) Immunofluorescence images showing GRK5 (red) and NFAT1 (green) staining in 381T cells, with overlay of both channels (yellow). (G) Proximity Ligation Assay (PLA) to assess GRK5-NFAT1 protein interaction in GRK5 wildtype (CTRL) and GRK5-knockout 381T cells. Red dots represent points of GRK5 and NFAT1 in close proximity. 
are thought to be the major drivers of cancer relapse and metastasis in selected cancer types [7, 38]. Disease relapse or metastasis of RMS carries a poor survival prognosis [3]. Identifying potential targets that regulate TPC survival could potentially provide a solution for treating
RMS disease relapse and metastasis. Our study shows that GRK5 loss-of-function significantly reduces the selfrenewal capacity of RMS cells in vitro and in vivo. The stem-like RMS spheres harboring GRK5 knockout show increased cell death. Our findings indicate that GRK5

A

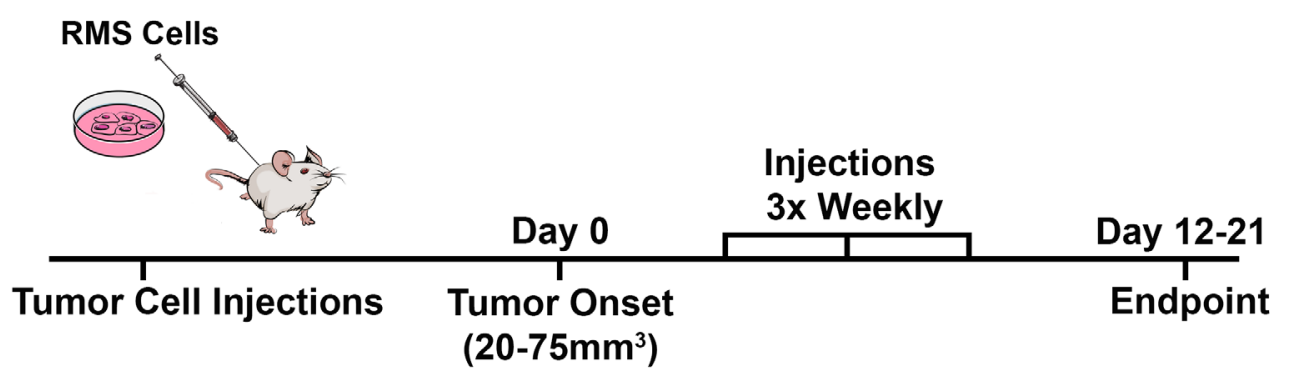

B

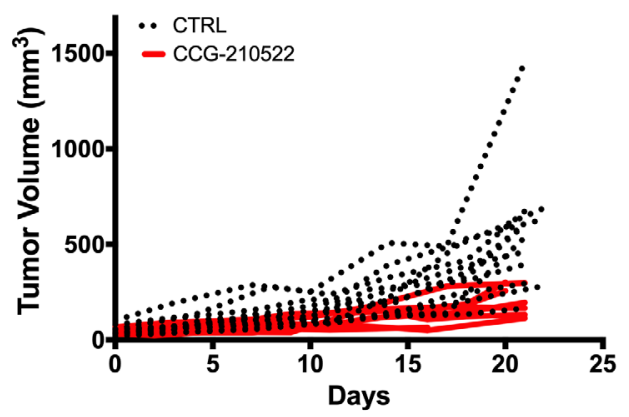

D

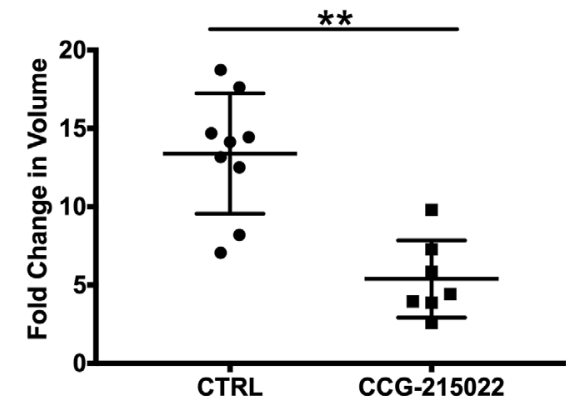

C

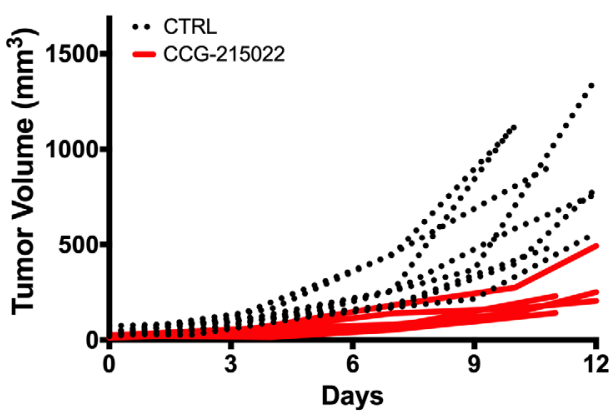

E

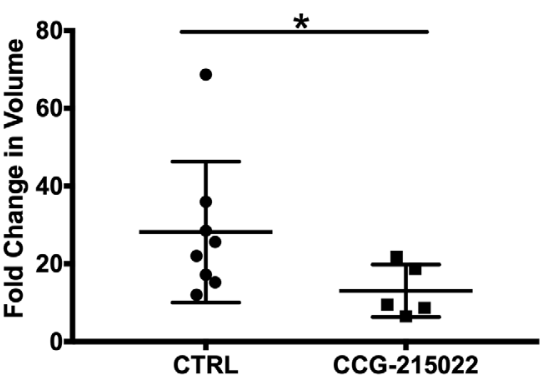

$\mathbf{F}$

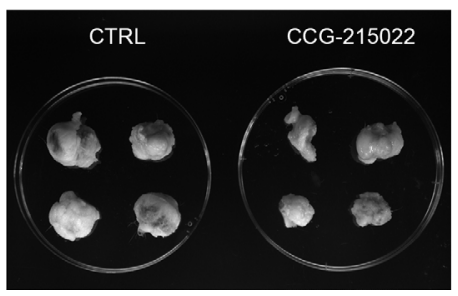

G

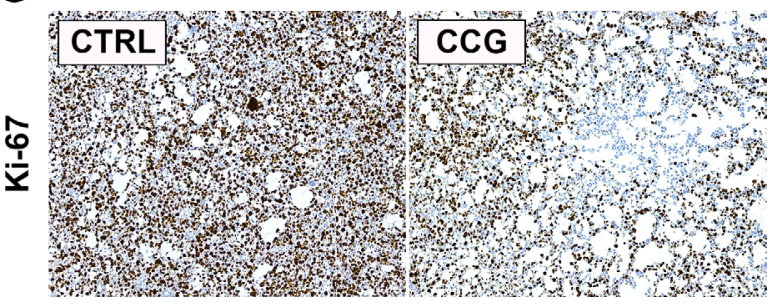

Figure 5: Treatment of RMS tumor with CCG-215022, a GRK5 inhibitor, reduces tumor growth in vivo. (A) Schematic of the RMS xenograft in NSG mice experiment. (B, C) Summary of 381T (B) and Rh5 (C) tumor growth during treatment period following CCG-215022 or DMSO control (CTRL) treatment. (D, E) Summary of 381T (D) and Rh5 (E) tumor volume fold change following CCG-215022 or DMSO control (CTRL) treatment. Each data point represents one mouse with error bars representing standard deviation. ${ }^{*}=p<0.05,{ }^{* *}=p<0.01$ (Mann-Whitney Statistical Test). (F) Representative images of Rh5 tumors harvested from DMSO vehicle control (CTRL) treated and CCG-215022 treated mice at the end of treatment period. (G) Representative images of Immunohistochemistry for Ki67 on DMSO control (CTRL) and CCG-215022 (CCG)-treated tumors. 
is a promising therapeutic target against RMS stemlike features. Further investigation is required to assess whether loss of GRK5 leads to reduced heterogeneity of RMS tumors and thereby reduces the potential for resistance against standard-of-care therapies.

Inhibitors selective to GRK5 are currently limited. Amlexanox, an FDA-approved anti-inflammatory drug, has been shown to inhibit GRK5 activity [39]. However, Amlexanox is a non-specific inhibitor with cross reactivity with other proteins and pathways such as IKBKE in the Hippo pathway [40]. CCG-215022, an investigational compound developed by John Tesmer's group at the University of Michigan, shows high selectivity against GRK5 [41]. In our study, treatment of both ERMS and ARMS xenograft tumors treated with CCG-215022 significantly reduces tumor growth. CCG-215022-bound GRK5 shows disorder in the residues pertaining to the $\mathrm{N}$-terminus, C-terminus and active site tether regions [41]. We show that a kinase deficient GRK5 mutant rescues the GRK5 loss-of-function growth phenotype. Based on this finding, it is possible that the functional domain of GRK5 that regulates RMS cell growth likely resides in one of these disordered regions. While additional testing to assess the toxicity profile of CCG-215022 in pre-clinical models is necessary, we have shown that inhibition of GRK5 is a promising therapeutic option for RMS patients.

With treatment options against RMS remaining relatively unchanged over last 3 decades, there remains a need for more effective therapeutic targets. From a comprehensive siRNA library screen against the human kinome, we have identified GRK5 as a novel regulator of both RMS self-renewal and cell growth. Our functional characterization of GRK5 in vitro and in vivo demonstrates that GRK5 regulates ERMS cell growth in a kinaseindependent manner and is essential for RMS self-renewal capacity. A GRK5 inhibitor, CCG-215022, recapitulates the loss-of-function effects of GRK5. Thus, our findings demonstrate the promise of GRK5 as a therapeutic target against RMS disease progression and relapse.

\section{MATERIALS AND METHODS}

\section{siRNA kinome library screen}

To identify potential candidate kinases that are important for the self-renewal of ERMS, we utilized the Quellos high-throughput screening core facility in the Institute of Stem Cell and Regenerative Medicine at the University of Washington to perform an siRNA library screen against the human kinome (714 kinases) in two different cell lines derived from ERMS (RD and $381 \mathrm{~T}$ ). Each cell line was transfected with a pool of 3 siRNAs against each kinase, along with control (scramble) siRNAs, in 384-well low attachment plates to induce sphere formation. The sphere assay was used as a surrogate in vitro assay for assessing the self-renewal capacity of tumor cell. The ATP-based Cell Titer Glo assay (Promega, Madison, WI) was performed on the siRNAtreated adherent cells, and high-content imaging was performed at the Quellos core facility at day 5 post-siRNA transfection.

\section{CRISPR/Cas9 gene targeting in human RMS cells}

Single gene knockout was accomplished using lentiviral transduction of RMS cells with Cas9 expressing and gene-specific double gRNA constructs. Lentiviral transduced cells were placed under antibiotic selection and plated for assays 7 days later. Cloning of Cas9 and gRNA expression constructs was performed as described previously [36]. Overexpression constructs used in GRK5 functional experiments were amplified from cDNA generated from the $381 T$ ERMS cell line. Silent mutations to PAM sites were introduced in the GRK5 overexpression constructs to generate Cas9 resistant GRK5 protein. RMS cells were then transduced with 3 separate lentiviruses; Cas9 virus, dgRNA virus and GRK5 WT/KD overexpression virus. Domain mutations made to GRK5 to generate wildtype (WT) and kinase dead (KD) variants was done using Gibson cloning based off of previous studies [25].

The following gRNAs were used for targeting genes in human RMS cell lines: GRK5: gRNA1GGACCTGGTCTCCCAGACGG; gRNA2- GGAGCAGC CCTTTCTTGGG; NFAT1: gRNA1- GACGGAGTGA TCTCGATCCG; gRNA2- GATCCCACAAGGCGAGT CCG; FES: gRNA1-GGCCGAGCTTCGTCTACTGG; gRNA2- GAGCCTGCTCATCCGGGAA; LTK: gRNA1GCTGGCTCCAAGATACTAGG; gRNA2- GACCAGCG TGGTGGTGACCG; LYN: gRNA1- GTAGCCTTGT ACCCCTATGA; gRNA2- GGAATGGCATACATCGAG; NME9: gRNA1- GACCTCGATCCTCATCTTC; gRNA2GATGTCCTCGAAAAGTACAG; $P I K 3 C 2 A$ : gRNA1GCACAGGTTTATAACAAGC; gRNA2 - GGGGCGCTT GCTAATATTTT.

\section{Cell-based assays}

RMS cell growth was assessed via cell counts or an ATP-based luminescent cell viability assay, Cell Titer Glo (Promega, Madison, WI). Myogenic differentiation was performed following serum starvation of RMS cells in $2 \%$ horse serum/DMEM for 72 hours prior to fixation in $2 \%$ paraformaldehyde. Immunofluorescence against MF20 (myosin heavy chain) was then performed. Self-renewal was assessed by visual counts of the spheroids, induced in growth factors (EGF, bFGF, PDGF-A, PDGF-B) enriched neurobasal medium as previous described [42]. Apoptosis was assessed using a flow-cytometry based assay using the Annexin V, Alexa Fluor 647 conjugate (Life Technologies, Carlsbad, CA). Cell cycle analysis was done with flow 
cytometry on cells pulsed with EdU for 2 hours using the Click-iT EdU Alexa Fluor 647 Flow Cytometry Assay kit (Life Technologies, Carlsbad, CA).

\section{Human xenografts and drug treatment}

All mouse experiments were approved by the University of Washington Subcommittee on Research Animal Care under IACUC protocol \#4330-01. 6-7 immunocompromised NOD-SCID IL2rg-/- (NSG) mice were xenografted via subcutaneous injections into the flanks with approximately 1-2 × $10^{6}$ RMS cells (RH5 or $381 \mathrm{~T}$ ) suspended in Matrigel. When the tumor size reached $20-75 \mathrm{~mm}^{3}$, CCG-215022 $(10 \mathrm{mg} / \mathrm{kg})$ or vehicle (DMSO) were given intraperitoneal every 3 days for up to 21 days or until tumors reached end point $\left(750 \mathrm{~mm}^{3}\right)$. Tumor measurements were made with calipers every 3-4 days at tumor onset until tumors reached end point or at the end of drug treatment, whichever came first. For limiting dilution experiments, 6 NSG mice were given RMS cell injections (Rh5 or 381T) of either control or GRK5 knock-out cells at either $2 \times 10^{3}, 1 \times 10^{4}, 5 \times 10^{4}$ dilutions in the same manner as previously described. Analysis of limiting dilution data was performed by the extreme limiting dilution (ELDA) software as previously described [43]. All mice were humanly euthanized for tumor tissue harvesting at the end of the experiment.

\section{Immunohistochemistry and immunofluorescence}

The RMS tissue microarray was obtained from Seattle Children's Hospital. Immunohistochemistry was performed at the Histology and Imaging core facility at the University of Washington. Immunofluorescence was performed as previously described [29]. Duolink Proximity Ligation Assay (PLA) by Sigma Aldrich was used to visualize GRK5-NFAT1 activity. The following antibodies were used for immunohistochemistry: rabbit polyclonal anti human mouse monoclonal anti human Ki-67 (MIB1) (1:100, Dako, Santa Clara, CA), GRK5 (N145/20) (1:100, Abcam, Cambridge, MA); for immunofluorescence: GRK5 (N145/20) (1:150, Abcam, Cambridge, MA), NFAT1 (D43B1) (1:100, Cell Signaling Technology, Danvers, MA).

\section{Western blots}

Cell lysates from RMS cells were counted and lysed in RIPA buffer (Thermo Fisher Scientifc, Waltham, MA) with protease inhibitors plus $2 \times$ sample buffer $(100 \mathrm{mM}$ Tris pH6.8, 4\%SDS, $20 \%$ glycerol). Equal amounts of protein lysates were electrophoresed on a $4-15 \%$ gradient SDS-polyacrylamide gel (BioRad, Hercules, CA) and fast transferred to Immun-Blot PVDF membranes (BioRad, Hercules, CA) using the Turbo-Blot Transfer system (BioRad, Hercules, CA) We used the following antibodies: GRK5 (N145/20) (1:100, Abcam, Cambridge, MA),
GAPDH (14C10) (1:2500, Cell Signaling Technology, Danvers, MA), Cleaved Caspase 3 (Asp175) (1:200, Cell Signaling Technology, Danvers, MA). Goat anti-mouse or anti-rabbit HRP conjugated IgG secondary antibodies were obtained from Santa Cruz Biotechnology (Dallas, TX). Membranes were blocked in 5\% milk in Tris Buffered Saline plus Tween (20mM Tris, $136 \mathrm{mM} \mathrm{NaCl}, 1 \%$ Tween 20, pH 7.4 TBST). Quantitative analysis of Western blot images was performed on ImageJ.

\section{Human expression data analysis}

RNA was collected from human cell lines lysates (myoblasts, NHDF, 381T, SMS-CTR, RH30, RH5) using Qiagen RNeasy Plus Mini Kit (Qiagen, Germantown, MD). cDNA was then generated using High Capacity cDNA Reverse Transcription Kit from Applied Biosystems (Foster City, CA). RT-PCR reactions were then run with iTaq Universal SYBR Green mix on a CFX Connect Real Time System (BioRad, Hercules, CA). RT-PCR primers used are listed below: GRK5 FWD - GTCTGTCCACGAGTACCTGA; REV - CAGG CATACATTTTACCCGT; NFAT1 FWD - ACGAGCTT GACTTCTCCACC; REV - TGCATTCGGCTCTTC TTCGT

\section{Statistics}

Mann-Whitney statistical test was run on drug treat RMS tumor mouse experiments to assess statistical significance in differences between experimental and control. Two tailed Student's t-test was applied when appropriate.

\section{ACKNOWLEDGMENTS AND FUNDING}

We would like to thank the Molecular Medicine and Mechanism of Disease (M3D) PhD program at the University of Washington School of Medicine for their support. Additional thanks to all the undergraduates and Chen Lab members who provided technical assistance to the study (Phuong Van, Shelly Lin, Henna Di, Dr. Michael Phelps, Maria Pavlova and Dr. Julia Sidorova). EYC is supported by NIH R01 CA196882.

\section{CONFLICTS OF INTEREST}

The authors declare no potential conflicts of interest.

\section{REFERENCES}

1. Shern JF, Chen L, Chmielecki J, Wei JS, Patidar R, Rosenberg M, Ambrogio L, Auclair D, Wang J, Song YK, Tolman C, Hurd L, Liao H, et al. Comprehensive genomic analysis of rhabdomyosarcoma reveals a landscape of alterations affecting a common genetic axis in 
fusion-positive and fusion-negative tumors. Cancer Discov. 2014; 4:216-31. https://doi.org/10.1158/2159-8290.CD-130639. [PubMed]

2. Barr FG, Galili N, Holick J, Biegel JA, Rovera G, Emanuel BS. Rearrangement of the PAX3 paired box gene in the paediatric solid tumour alveolar rhabdomyosarcoma. Nat Genet. 1993; 3:113-17. https://doi.org/10.1038/ng0293-113. [PubMed]

3. Pappo AS, Anderson JR, Crist WM, Wharam MD, Breitfeld PP, Hawkins D, Raney RB, Womer RB, Parham DM, Qualman SJ, Grier HE. Survival after relapse in children and adolescents with rhabdomyosarcoma: A report from the Intergroup Rhabdomyosarcoma Study Group. J Clin Oncol. 1999; 17:3487-93. https://doi.org/10.1200/ JCO.1999.17.11.3487. [PubMed]

4. Kaur G, Kim J, Kaur R, Tan I, Bloch O, Sun MZ, Safaee M, Oh MC, Sughrue M, Phillips J, Parsa AT. G-protein coupled receptor kinase (GRK)-5 regulates proliferation of glioblastoma-derived stem cells. J Clin Neurosci. 2013; 20:1014-18. https://doi.org/10.1016/j.jocn.2012.10.008. [PubMed]

5. Lawson DA, Bhakta NR, Kessenbrock K, Prummel KD, Yu Y, Takai K, Zhou A, Eyob H, Balakrishnan S, Wang CY, Yaswen P, Goga A, Werb Z. Single-cell analysis reveals a stem-cell program in human metastatic breast cancer cells. Nature. 2015; 526:131-35. https://doi.org/10.1038/ nature15260. [PubMed]

6. Jahchan NS, Lim JS, Bola B, Morris K, Seitz G, Tran KQ, Xu L, Trapani F, Morrow CJ, Cristea S, Coles GL, Yang D, Vaka D, et al. Identification and targeting of longterm tumor-propagating cells in small cell lung cancer. Cell Rep. 2016; 16:644-56. https://doi.org/10.1016/j. celrep.2016.06.021. [PubMed]

7. Kreso A, Dick JE. Evolution of the cancer stem cell model. Cell Stem Cell. 2014; 14:275-91. https://doi.org/10.1016/j. stem.2014.02.006. [PubMed]

8. Ignatius MS, Chen E, Elpek NM, Fuller AZ, Tenente IM, Clagg R, Liu S, Blackburn JS, Linardic CM, Rosenberg AE, Nielsen PG, Mempel TR, Langenau DM. In vivo imaging of tumor-propagating cells, regional tumor heterogeneity, and dynamic cell movements in embryonal rhabdomyosarcoma. Cancer Cell. 2012; 21:680-93. https://doi.org/10.1016/j. ccr.2012.03.043. [PubMed]

9. Walter D, Satheesha S, Albrecht P, Bornhauser BC, D'Alessandro V, Oesch SM, Rehrauer H, Leuschner I, Koscielniak E, Gengler C, Moch H, Bernasconi M, Niggli FK, et al. CD133 positive embryonal rhabdomyosarcoma stem-like cell population is enriched in rhabdospheres. PLoS One. 2011; 6:e19506. https://doi. org/10.1371/journal.pone.0019506. [PubMed]

10. Gross S, Rahal R, Stransky N, Lengauer C, Hoeflich KP. Targeting cancer with kinase inhibitors. J Clin Invest. 2015; 125:1780-89. https://doi.org/10.1172/JCI76094. [PubMed]
11. Hanahan D, Weinberg RA. Hallmarks of cancer: the next generation. Cell. 2011; 144:646-74. https://doi. org/10.1016/j.cell.2011.02.013. [ [PubMed]

12. Roskoski R Jr. Properties of FDA-approved small molecule protein kinase inhibitors. Pharmacol Res. 2019; 144:19-50. https://doi.org/10.1016/j.phrs.2019.03.006. [PubMed]

13. Yohe ME, Gryder BE, Shern JF, Song YK, Chou HC, Sindiri S, Mendoza A, Patidar R, Zhang X, Guha R, Butcher D, Isanogle KA, Robinson CM, et al. MEK inhibition induces MYOG and remodels super-enhancers in RAS-driven rhabdomyosarcoma. Sci Transl Med. 2018; 10:eaan4470. https://doi.org/10.1126/scitranslmed. aan4470. [PubMed]

14. Stewart E, McEvoy J, Wang H, Chen X, Honnell V, Ocarz M, Gordon B, Dapper J, Blankenship K, Yang Y, Li Y, Shaw TI, Cho JH, et al. Identification of Therapeutic Targets in Rhabdomyosarcoma through Integrated Genomic, Epigenomic, and Proteomic Analyses. Cancer Cell. 2018; 34:411-426.e19. https://doi.org/10.1016/j. ccell.2018.07.012. [PubMed]

15. Chen EY, DeRan MT, Ignatius MS, Grandinetti KB, Clagg R, McCarthy KM, Lobbardi RM, Brockmann J, Keller C, Wu X, Langenau DM. Glycogen synthase kinase 3 inhibitors induce the canonical WNT/ $\beta$-catenin pathway to suppress growth and self-renewal in embryonal rhabdomyosarcoma. Proc Natl Acad Sci U S A. 2014; 111:5349-54. https://doi.org/10.1073/pnas.1317731111. [PubMed]

16. Willets JM, Challiss RA, Nahorski SR. Non-visual GRKs: are we seeing the whole picture? Trends Pharmacol Sci. 2003; 24:626-33. https://doi.org/10.1016/j.tips.2003.10. 003. [PubMed]

17. Islam KN, Bae JW, Gao E, Koch WJ. Regulation of nuclear factor $\kappa \mathrm{B}(\mathrm{NF}-\kappa \mathrm{B})$ in the nucleus of cardiomyocytes by $\mathrm{G}$ protein-coupled receptor kinase 5 (GRK5). J Biol Chem. 2013; 288:35683-89. https://doi.org/10.1074/jbc. M113.529347. [PubMed]

18. Philipp M, Berger IM, Just S, Caron MG. Overlapping and opposing functions of $\mathrm{G}$ protein-coupled receptor kinase 2 (GRK2) and GRK5 during heart development. J Biol Chem. 2014; 289:26119-30. https://doi.org/10.1074/jbc. M114.551952. [PubMed]

19. Zhang Y, Matkovich SJ, Duan X, Gold JI, Koch WJ, Dorn GW. Nuclear Effects of GRK5 on HDAC5-regulated Gene Transcription in Heart Failure. Circ Heart Fail. 2011; 4:659-68. https://doi.org/10.1161/CIRCHEART FAILURE.111.962563. [PubMed]

20. Hullmann JE, Grisanti LA, Makarewich CA, Gao E, Gold JI, Chuprun JK, Tilley DG, Houser SR, Koch WJ. GRK5-mediated exacerbation of pathological cardiac hypertrophy involves facilitation of nuclear NFAT activity. Circ Res. 2014; 115:976-85. https://doi.org/10.1161/ CIRCRESAHA.116.304475. [PubMed] 
21. Lessel D, Muhammad T, Casar Tena T, Moepps B, Burkhalter MD, Hitz MP, Toka O, Rentzsch A, Schubert S, Schalinski A, Bauer UM, Kubisch C, Ware SM, Philipp $\mathrm{M}$. The analysis of heterotaxy patients reveals new loss-offunction variants of GRK5. Sci Rep. 2016; 6:33231. https:// doi.org/10.1038/srep33231. [PubMed]

22. Martini JS, Raake P, Vinge LE, DeGeorge BR Jr, Chuprun JK, Harris DM, Gao E, Eckhart AD, Pitcher JA, Koch WJ. Uncovering G protein-coupled receptor kinase-5 as a histone deacetylase kinase in the nucleus of cardiomyocytes. Proc Natl Acad Sci U S A. 2008; 105:12457-62. https://doi.org/10.1073/pnas.0803153105. [PubMed]

23. Jiang LP, Fan SQ, Xiong QX, Zhou YC, Yang ZZ, Li GF, Huang YC, Wu MG, Shen QS, Liu K, Yang CP, Chen YB. GRK5 functions as an oncogenic factor in non-smallcell lung cancer. Cell Death Dis. 2018; 9:295. https://doi. org/10.1038/s41419-018-0299-1. [PubMed]

24. Kim JI, Chakraborty P, Wang Z, Daaka Y. G-protein coupled receptor kinase 5 regulates prostate tumor growth. J Urol. 2012; 187:322-29. https://doi.org/10.1016/j. juro.2011.09.049. [PubMed]

25. Johnson LR, Robinson JD, Lester KN, Pitcher JA. Distinct structural features of $\mathrm{G}$ protein-coupled receptor kinase 5 (GRK5) regulate its nuclear localization and DNA-binding ability. PLoS One. 2013; 8:e62508. https://doi.org/10.1371/ journal.pone.0062508. [PubMed]

26. Sorriento D, Santulli G, Ciccarelli M, Maione AS, Illario M, Trimarco B, Iaccarino G. The Amino-Terminal Domain of GRK5 Inhibits Cardiac Hypertrophy through the Regulation of Calcium-Calmodulin Dependent Transcription Factors. Int J Mol Sci. 2018; 19:E861. https://doi.org/10.3390/ ijms19030861. [PubMed]

27. Macian F. NFAT proteins: key regulators of T-cell development and function. Nat Rev Immunol. 2005; 5:47284. https://doi.org/10.1038/nri1632. [PubMed]

28. Pastrana E, Silva-Vargas V, Doetsch F. Eyes wide open: a critical review of sphere-formation as an assay for stem cells. Cell Stem Cell. 2011; 8:486-98. https://doi. org/10.1016/j.stem.2011.04.007. [PubMed]

29. Phelps MP, Bailey JN, Vleeshouwer-Neumann T, Chen EY. CRISPR screen identifies the NCOR/HDAC3 complex as a major suppressor of differentiation in rhabdomyosarcoma. Proc Natl Acad Sci U S A. 2016; 113:15090-95. https://doi. org/10.1073/pnas.1610270114. [PubMed]

30. Watari K, Nakaya M, Kurose H. Multiple functions of G protein-coupled receptor kinases. J Mol Signal. 2014; 9:1. https://doi.org/10.1186/1750-2187-9-1. [PubMed]

31. Baumgart S, Glesel E, Singh G, Chen NM, Reutlinger K, Zhang J, Billadeau DD, Fernandez-Zapico ME, Gress TM, Singh SK, Ellenrieder V. Restricted heterochromatin formation links NFATc2 repressor activity with growth promotion in pancreatic cancer. Gastroenterology. 2012; 142:388-98.e1-7. https://doi.org/10.1053/j.gastro.2011. 11.001. [PubMed]
32. Baumgart S, Chen NM, Siveke JT, König A, Zhang JS, Singh SK, Wolf E, Bartkuhn M, Esposito I, Heßmann E, Reinecke J, Nikorowitsch J, Brunner M, et al. Inflammationinduced NFATc1-STAT3 transcription complex promotes pancreatic cancer initiation by KrasG12D. Cancer Discov. 2014; 4:688-701. https://doi.org/10.1158/2159-8290. CD-13-0593. [PubMed]

33. Chen X, Zhu H, Yuan M, Fu J, Zhou Y, Ma L. G-proteincoupled receptor kinase 5 phosphorylates p53 and inhibits DNA damage-induced apoptosis. J Biol Chem. 2010; 285:12823-30. https://doi.org/10.1074/jbc.M109.094243. [PubMed]

34. Michal AM, So CH, Beeharry N, Shankar H, Mashayekhi R, Yen TJ, Benovic JL. G Protein-coupled receptor kinase 5 is localized to centrosomes and regulates cell cycle progression. J Biol Chem. 2012; 287:6928-40. https://doi. org/10.1074/jbc.M111.298034. [PubMed]

35. Ognjanovic S, Martel G, Manivel C, Olivier M, Langer E, Hainaut P. Low Prevalence of TP53 Mutations and MDM2 Amplifications in Pediatric Rhabdomyosarcoma. Sarcoma. 2012; 2012:492086. https://doi.org/10.1155/2012/492086. [PubMed]

36. Hinson AR, Jones R, Crose LE, Belyea BC, Barr FG, Linardic CM. Human rhabdomyosarcoma cell lines for rhabdomyosarcoma research: utility and pitfalls. Front Oncol. 2013; 3:183. https://doi.org/10.3389/ fonc.2013.00183. [PubMed]

37. Mognol GP, Carneiro FR, Robbs BK, Faget DV, Viola JP. Cell cycle and apoptosis regulation by NFAT transcription factors: new roles for an old player. Cell Death Dis. 2016; 7:e2199. https://doi.org/10.1038/cddis.2016.97. [PubMed]

38. Saygin C, Matei D, Majeti R, Reizes O, Lathia JD. Targeting Cancer Stemness in the Clinic: From Hype to Hope. Cell Stem Cell. 2019; 24:25-40. https://doi.org/10.1016/j. stem.2018.11.017. [PubMed]

39. Homan KT, Wu E, Cannavo A, Koch WJ, Tesmer JJ. Identification and characterization of amlexanox as a $\mathrm{G}$ protein-coupled receptor kinase 5 inhibitor. Molecules. 2014; 19:16937-49. https://doi.org/10.3390/ molecules191016937. [PubMed]

40. Liu Y, Lu J, Zhang Z, Zhu L, Dong S, Guo G, Li R, Nan Y, Yu K, Zhong Y, Huang Q. Amlexanox, a selective inhibitor of IKBKE, generates anti-tumoral effects by disrupting the Hippo pathway in human glioblastoma cell lines. Cell Death Dis. 2017; 8:e3022. https://doi.org/10.1038/cddis.2017.396. [PubMed]

41. Homan KT, Waldschmidt HV, Glukhova A, Cannavo A, Song J, Cheung JY, Koch WJ, Larsen SD, Tesmer JJ. Crystal Structure of G Protein-coupled Receptor Kinase 5 in Complex with a Rationally Designed Inhibitor. J Biol Chem. 2015; 290:20649-59. https://doi.org/10.1074/jbc. M115.647370. [PubMed]

42. Vleeshouwer-Neumann T, Phelps M, Bammler TK, MacDonald JW, Jenkins I, Chen EY. Histone Deacetylase 
Inhibitors Antagonize Distinct Pathways to Suppress Tumorigenesis of Embryonal Rhabdomyosarcoma. PLoS One. 2015; 10:e0144320. https://doi.org/10.1371/journal. pone.0144320. [ $\underline{\text { PubMed] }}$
43. Hu Y, Smyth GK. ELDA: extreme limiting dilution analysis for comparing depleted and enriched populations in stem cell and other assays. J Immunol Methods. 2009; 347:70-78. https://doi.org/10.1016/j.jim.2009.06.008. [PubMed] 